\title{
Rationale for Medium Cutoff Membranes in COVID-19 Patients Requiring Renal Replacement Therapy
}

\author{
Claudio Ronco ${ }^{a, b}$ Thiago Reis ${ }^{b, c}$ Mario Cozzolino d,e \\ aDepartment of Medicine (DIMED), University of Padova, Padova, Italy; ${ }^{\mathrm{b}}$ Department of Nephrology, \\ Dialysis and Transplantation, International Renal Research Institute of Vicenza, San Bortolo Hospital, Vicenza, Italy; \\ 'Department of Nephrology, Clínica de Doenças Renais de Brasília, Brasília, Brazil; dDepartment of Health Sciences, \\ University of Milan, Milan, Italy; ${ }^{~}$ Department of Nephrology and Dialysis, ASST Santi Paolo e Carlo, Milan, Italy
}

\section{Keywords}

Coronavirus disease $2019 \cdot$ Dialysis membranes ·

Hemodialysis · Infection · Medium cutoff dialyzer ·

Hemoadsorption

\begin{abstract}
The current pandemic of coronavirus disease 2019 (COVID-19) spotlighted the vulnerability of patients with chronic kidney disease stage 5 on maintenance hemodialysis (HD) to the viral infection. Social distancing is the most effective preventive measure to reduce the risk of infection. Nonetheless, the necessity to frequently reach the dialysis center and the inherent social gathering both impede social distancing and also self-quarantine for infected individuals. A baseline hyperinflammatory state driven by factors such as the retention of uremic toxins afflicts these patients. Concomitantly, a condition of relative immunosuppression is also attributed to similar factors. The use of high-flux (HF) dialyzers for HD is the standard of care. However, with HF membranes, the removal of large middle molecules is scant. Medium cutoff (MCO) dialyzers are a new class of membranes that allow substantial removal of large middle molecules with negligible albumin losses. Recent trials confirmed long-term safety
\end{abstract}

and long-term sustained reduction in the concentration of large uremic toxins with MCO dialyzers. Herein, we discuss the rationale for applying MCO membranes in COVID-19 patients and its possible immunoadjuvant effects that could mitigate the burden of COVID-19 infection in dialysis patients. We also discuss the direct cytopathic effect of the virus on renal tissue and extracorporeal blood purification techniques that can prevent kidney damage or reduce acute kidney injury progression.

(c) 2020 S. Karger AG, Basel

\section{COVID-19 Pandemic and the Kidney}

Since the new COVID-19 outbreak has been declared a pandemic, interesting epidemiological and pathophysiological data are emerging $[1,2]$. In particular, the prevalence of kidney involvement among infected patients is difficult to establish due to differences in swab test policies. Nevertheless, a common scenario takes place with two main aspects related to the COVID-19 infection. The first is the direct acute kidney injury (AKI) occurring in patients admitted to the intensive care unit (ICU). Approximately $14 \%$ of individuals hospitalized for symp-

$\begin{aligned} & \text { karger@karger.com } \\ & \text { www.karger.com/nef }\end{aligned}$
Karger ${ }^{\prime /}$

Claudio Ronco

Department of Nephrology, Dialysis and Transplantation, San Bortolo Hospital Viale Rodolfi, 37

IT-36100 Vicenza (Italy)

cronco@goldnet.it 
tomatic COVID-19 infection require ICU admission [3] and $37 \%$ develop different stages of AKI. Of those with AKI, $14 \%$ required renal replacement therapy, meaning that $5 \%$ of all patients hospitalized with COVID-19 required some form of dialysis support [4]. In a prospective cohort study, 44\% of COVID-19 hospitalized patients presented proteinuria and 27\% hematuria [5]. The second aspect relates to chronic kidney disease (CKD) patients who might develop worsening of renal function or are already on some form of chronic dialysis treatment [6-11].

\section{Pathophysiology of Kidney Involvement}

After the lungs, the kidneys seem to be the most afflicted solid organ. SARS-CoV-2 viral load was found in all kidney compartments, mainly in glomerular cells [12]. Histopathological postmortem findings in 26 patients showed acute tubular necrosis as the most prevalent finding in COVID-19 patients [5], this was corroborated in a case series from ten patients with AKI that underwent native kidney needle biopsy [13]. Other case reports demonstrated viral copies in the cytoplasm of tubular cells [14]. In some infected individuals, the proximal tubule increases the expression of angiotensin-converting enzyme 2 (ACE2). The enzyme is present in high concentrations in the brush border of epithelial cells of the tubules. ACE2 acts as a receptor for SARS-CoV-2 spike protein, through which the virus gains access to the host cell [14]. Of note, ACE2 is also present in podocytes [15]. Additionally, viral particles were visualized by electron microscopy in podocytes $[15,16]$ and glomerular endothelial cells [17]. Proteinuria is not among the criteria for diagnosis of AKI according to current definitions [18]. Notwithstanding, proteinuria per se is a marker of kidney damage. The ubiquitous presence of the virus with the invasion of glomerular endothelial cells, podocytes, and tubular cells could explain the high prevalence of proteinuria. Damaged endothelial cells and disruption of the podocyte network both increase glomerular protein leakage, overwhelming the proximal tubules' capacity of protein reabsorption. Moreover, infected tubular cells might also be dysfunctional. We hypothesize that if biomarkers of tubular injury, such as neutrophil gelatinase-associated lipocalin (NGAL), and of cell-cycle arrest such as tissue inhibitor of metalloproteinase-2 (TIMP2) and insulin-like growth factor-binding protein 7 (IGFBP7) [18] were incorporated into clinical practice, the prevalence of AKI, considering also subclinical AKI, would sharply increase in COVID-19 patients. Our group published the first study evaluating the product [TIMP-2]•[IGFBP7] in 23 hospitalized patients with SARS-CoV-2 infection. In this ongoing observational study there were no clear differences between trends in [TIMP-2]•[IGFBP7] of AKI versus non-AKI at admission. However, all patients that progressed to stage $3 \mathrm{AKI}$ had [TIMP-2]•[IGFBP7] levels $>2(\mathrm{ng} / \mathrm{mL})^{2} / 1,000$, and all AKI patients with [TIMP-2]•[IGFBP7] levels $\leq 0.3$ $(\mathrm{ng} / \mathrm{mL})^{2} / 1,000$ did not experience progression of their AKI stage [19]. We are currently analyzing our data on the evaluation of urinary NGAL, a biomarker of tubular cell injury.

The ICU patient might also develop AKI as a final common pathway of systemic inflammation with augmented immune response leading to unrestrained circulating levels of pro-inflammatory mediators and direct cytokine-induced organ damage [20-24]. In general, patients display a hyperinflammatory state that should be considered as a target for different therapeutic options, including pharmacological [21-24] and extracorporeal $[2,25]$ cytokine blockade.

Ambulatory care patients with advanced CKD and/or undergoing chronic hemodialysis (HD) or peritoneal dialysis have 2 different pathways.

1. Some require ICU admission and need prolonged intermittent HD or continuous renal replacement therapy (CRRT).

2. Others need to continue their chronic dialysis regimen with specific isolation and possibly modified prescription based on metabolic and clinical demand [6-11].

\section{Extracorporeal Techniques for COVID-19 Kidney Patients}

Different approaches should be proposed for different indications and different patients. Patients with a systemic inflammatory syndrome, hemodynamic instability, need for vasopressors, and high serum concentrations of cytokines may represent an indication for hemoadsorption treatment with specific sorbents for few hours in the immediate phase and the subsequent use of CRRT to sustain removal of fluid, metabolites, and inflammatory mediators for hours or days [2, 25]. In a recent case report, Berlot et al. [26] applied both tocilizumab, an interleukin (IL)-6 antagonist, and hemoperfusion with a neutromacroporous sorbent cartridge in a severely ill patient with COVID-19 pneumonia and acute respiratory distress syndrome. The patient had clinical and laboratory 
signs of a hyperinflammatory state, and after hemoperfusion therapy ( 1 session per day in 3 consecutive days), there was a marked improvement in the respiratory index and chest radiography, along with reductions in IL- 6 and $\mathrm{C}$-reactive protein. The patient was extubated after 5 days and survived hospitalization. Of note, this patient was not on renal replacement therapy, illustrating that extracorporeal blood purification techniques can be applied even in the absence of kidney injury to prevent organ damage and mitigate organ failure. Other extracorporeal blood purification techniques that can be used in COVID-19 patients to remove inflammatory molecules are as follows:

1. Therapeutic plasma exchange.

2. Adsorptive cartridges containing polystyrene fibers functionalized with polymyxin-B, aiming endotoxin removal in patients with superimposed Gram-negative sepsis.

3. Adsorptive CRRT membranes such as acrylonitrile and sodium methallyl sulfonate plus polyethylenimine (AN69-PEI); surface-treated AN69; or polymethyl methacrylate.

4. Medium cutoff (MCO) or high cutoff HD membranes which can remove inflammatory mediators by means of diffusion and convection [25].

In patients with stage $5 \mathrm{CKD}$ on maintenance $\mathrm{HD}$ treatment, extra removal of pro-inflammatory mediators may also represent a logical approach.

\section{Rationale for MCO Membrane Application in Chronic HD Patients}

Recent reports have elucidated the removal mechanism of large middle molecular weight solutes including cytokines with the application of MCO membranes. In particular, this has been studied in chronic treatments defined as expanded HD [27]. MCO membranes present a significantly improved sieving curve compared to highflux (HF) membranes and a high retention onset value. At the same time, they display a significantly lower albumin loss than high cutoff membranes, allowing for safe and long-term applications [28]. These characteristics represent a phenomenal compromise to obtain the desired therapeutic target in COVID-19 patients both for chronic dialysis continuation and for application in CRRT. Although MCO dialyzers have not been indicated for CRRT in acute patients, we have personally tested the safe application with negligible albumin loss and improved cytokine clearance.

\section{Clinical Experience with MCO Membrane}

The retention of a large number of solutes that are normally excreted or metabolized by the kidney is responsible for the classical symptoms in HD patients. These molecules are defined as uremic toxins and can be classified into 3 groups: small water-soluble molecules, middle molecules, and protein-bound toxins. The MCO dialyzer allows the removal of middle molecules up to approximately 50,000 Da [29, 30].

We performed a prospective, open-label, controlled, cross-over study comparing the MCO dialyzer versus HF dialyzer in 20 patients [31]. Initially, 10 patients underwent conventional HF-HD and 10 patients underwent conventional MCO-HD for 3 months. Later, the patients switched and received the other treatment for a further 3 months. We demonstrated decreases in the levels of IL- $1 \beta$ and IL- 6 among patients receiving MCO-HD compared to those receiving conventional HF-HD. We also found a reduction in the incidence of infections during the MCOHD phase. Although difficult to interpret, because of the small sample size and potential bias, this result is very encouraging since infectious diseases are highly common in HD patients. These patients are more prone to infections with worse outcomes than the general population, mainly because of the high prevalence of diabetes mellitus, the presence of an indwelling catheter, and a condition of acquired immune dysfunction due to the retention of uremic toxins and chronic inflammation [32]. In accordance, a retrospective analysis comparing 49 chronic dialytic patients hospitalized with COVID-19 pneumonia versus 52 non-CKD patients hospitalized with COVID-19 pneumonia showed that chronic HD patients had lower lymphocyte counts, more abnormalities seen in chest computed tomography, higher incidence of shock, acute respiratory distress syndrome, and higher mortality risk [33].

We can propose different pathways through which MCO-HD could play a role in reducing the rate of severe infections. One of these is that treatment with MCO membranes may reduce inflammation, as supported by the lowering of the concentration of inflammatory cytokines and the reduction of transcription of pro-inflammatory cytokines in peripheral leukocytes [34]. Another possible explanation for the decrease of infectious events could be that MCO-HD reduces serum concentration of free light chains (FLC) since their retention in CKD patients is associated with in vitro inhibition of leukocyte chemotaxis [29].

Uniformly with our findings, two other recent trials demonstrated a reduction in the concentration of FLC [28, 
35]. One of the studies was similar in design to ours: a randomized, open-label, cross-over study, with 40 patients who were divided to carry out 3 months of MCO-HD followed by 3 months of HF-HD, or vice versa [35]. The primary outcome was myoglobin $(17 \mathrm{kDa})$ reduction rate (RR) after 3 months of MCO-HD compared to HF-HD. The RR for any given solute equals the subtraction of the pre-session from the post-session concentration, divided by the pre-session concentration. A statistically significant increase in myoglobin RR was found during the MCO dialyzer intervention period. However, the pre-dialysis concentration of myoglobin was similar in both groups, probably due to a rebound effect and redistribution of myoglobin into the blood compartment. Secondary outcomes included RR and pre-dialysis serum concentration of other middle molecules such as $\beta 2$-microglobulin $(11.8 \mathrm{kDa})$, tumor necrosis factor-alpha $(17.2 \mathrm{kDa})$, IL-6 $(21 \mathrm{kDa})$, kappa FLC $(22 \mathrm{kDa})$, prolactin $(23 \mathrm{kDa})$, and lambda FLC (45 kDa). Importantly, not only was the RR of $\beta 2$-microglobulin and FLC higher with the MCO dialyzer, but the pre-dialysis serum concentrations of $\beta 2$ microglobulin and FLC were also lower during the MCOHD phase, demonstrating that the effects of higher removal of middle molecules were sustained until the next dialytic treatment. The extrapolation of these results to cytokine removal based on their molecular weight is too simplistic. Accordingly, there was no difference in RR and pre-dialysis serum concentration for tumor necrosis factor-alpha $(17.2 \mathrm{kDa})$ and IL-6 $(21 \mathrm{kDa})$, irrespective of the dialytic membrane utilized. The virtual molecular radius, electrical charges, and protein binding are other variables that impact on solute clearance [36].

The other study, REMOVAL-HD [28], was a multicenter, open-label, non-randomized, single-arm trial with 89 individuals assigned to receive 4 weeks of HF-HD as wash-in, then 24 weeks of MCO-HD, and finally 4 weeks of HF-HD as wash-out. The primary endpoint was change in pre-dialysis serum albumin concentration before and after 6 months of MCO-HD. There was no difference in albumin concentration during the trial visits. After 2 weeks on MCO-HD, pre-dialysis serum concentrations of FLC were reduced, and this effect was maintained during the following 22 weeks of application of MCO membrane. The return to baseline values occurred 2 weeks after the return to HF-HD. These results cannot be generalized to all HD patients since malnourished patients and those with poor vascular access (blood flow $<300 \mathrm{~mL} / \mathrm{min}$ ) were ineligible for the study.

As an innovative treatment, the efficacy and safety of MCO-HD should be proven. The primary concern about safety mainly related to albumin losses has been overcome. Higher removal of middle molecules has been consistently seen in recent trials $[28,31,35]$. The application of MCO membranes in patients currently undergoing HF-HD could potentially reduce the incidence of infections. Moreover, in the COVID-19 outbreak, the higher clearance of FLC and some inflammatory cytokines may reduce the progression of mild presentations to moderate or severe clinical pictures in HD patients with COVID-19.

\section{Conclusion}

Our understanding of kidney involvement in COVID-19 has changed substantially in the past months. The renal tropism of SARS-CoV-2 is frequent [12]. The direct kidney invasion, as well as a hyperinflammatory state, might cause kidney damage $[1,2]$. Extracorporeal techniques can be helpful in the modulation of an immune dysregulated response, both in the severely ill and in the chronic HD patient. Recent clinical trials demonstrated consistent results regarding the application of MCO membranes in patients on maintenance $\operatorname{HD}[28,31,35]$. These trials confirmed long-term safety and long-term sustained reductions in the concentrations of large uremic toxins, cytokines, and FLC with MCO-HD when compared to conventional HF-HD. In the context of the COVID-19 pandemic, we presented the rationale that supports the use of MCO dialyzers in the chronic dialysis regimen. This strategy has been used in many dialysis facilities across Italy with the scope to prevent severe presentations of COVID-19. We aim to share our insights with the nephrology community and foster future collaborations between centers that will be fruitful to create new scientific evidence.

\section{Acknowledgement}

There are no acknowledgments to declare.

\section{Conflict of Interest Statement}

C.R. has been a consultant or advisory board member for ASAHI, Baxter, bioMérieux, B. Braun, CytoSorbents, Estor, Fresenius Medical Care, General Electric, Medtronic, and Toray in the past 3 years. T.R. has been a consultant or advisory board member for Baxter and B. Braun in the past 3 years. M.C. has been a consultant or advisory board member for Baxter in the past 3 years. 


\section{Funding Sources}

There are no funding sources to declare.

\section{Author Contributions}

All authors contributed equally to the creation, writing, and revision of the manuscript and approved submission.

\section{References}

1 Perico L, Benigni A, Remuzzi G. Should COVID-19 concern nephrologists? Why and to what extent? The emerging impasse of angiotensin blockade. Nephron. 2020;144(5):21321.

2 Ronco C, Reis T. Kidney involvement in COVID-19 and rationale for extracorporeal therapies. Nat Rev Nephrol. 2020;16(6):308-10.

3 Richardson S, Hirsch JS, Narasimhan M, Crawford JM, McGinn T, Davidson KW, et al. Presenting characteristics, comorbidities, and outcomes among 5,700 patients hospitalized with COVID-19 in the New York City area. JAMA. 2020 Apr;323(20):2052-59.

4 Hirsch JS, Ng JH, Ross DW, Sharma P, Shah $\mathrm{HH}$, Barnett RL, et al. Acute kidney injury in patients hospitalized with COVID-19. Kidney Int. 2020 May.

5 Su H, Yang M, Wan C, Yi L-X, Tang F, Zhu $\mathrm{H}-\mathrm{Y}$, et al. Renal histopathological analysis of 26 postmortem findings of patients with $\mathrm{CO}$ VID-19 in China. Kidney Int. 2020 Apr;98(1): 219-227.

6 Basile C, Combe C, Pizzarelli F, Covic A, Davenport A, Kanbay M, et al. Recommendations for the prevention, mitigation and containment of the emerging SARS-CoV-2 (COVID-19) pandemic in haemodialysis centres. Nephrol Dial Transpl. 2020 Mar;35(5):73741.

7 Meijers B, Messa P, Ronco C. Safeguarding the maintenance hemodialysis patient population during the coronavirus disease 19 pandemic. Blood Purif. 2020 Apr;49:259-64.

8 Kliger AS, Silberzweig J. Mitigating risk of COVID-19 in dialysis facilities. Clin J Am Soc Nephrol. 2020 May;15(5):707-9.

9 Kliger AS, Cozzolino M, Jha V, Harbert G, Ikizler TA. Managing the COVID-19 pandemic: international comparisons in dialysis patients. Kidney Int. 2020;98(1):12-6.

10 Alberici F, Delbarba E, Manenti C, Econimo L, Valerio F, Pola A, et al. A report from the Brescia renal COVID task force on the clinical characteristics and short-term outcome of hemodialysis patients with SARS-CoV-2 infection. Kidney Int. 2020 Jul;98(1):20-6.

11 Cozzolino M, Piccoli GB, Ikizler TA, Ronco C. The COVID-19 infection in dialysis: are home-based renal replacement therapies a way to improve patient management? J Nephrol. 2020 Jun.

12 Puelles VG, Lütgehetmann M, Lindenmeyer MT, Sperhake JP, Wong MN, Allweiss L, et al. Multiorgan and renal tropism of SARSCoV-2. N Engl J Med. 2020 May.
13 Sharma P. COVID-19-Associated Kidney Injury: A Case Series of Kidney Biopsy Findings. J Am Soc Nephrol. 2020. 10.1681/ASN. 2020050699.

14 Farkash EA, Wilson AM, Jentzen JM. Ultrastructural evidence for direct renal infection with SARS-CoV-2. J Am Soc Nephrol. 2020 May;ASN.2020040432.

15 Kissling S, Rotman S, Gerber C, Halfon M, Lamoth F, Comte D, et al. Collapsing glomerulopathy in a COVID-19 patient. Kidney Int. 2020 Jul;98(1):228-31.

16 Abbate M, Rottoli D, Gianatti A. COVID-19 attacks the kidney: ultrastructural evidence for the presence of virus in the glomerular epithelium. Nephron. 2020 Jun;144(7):341-2.

17 Varga Z, Flammer AJ, Steiger P, Haberecker $M$, Andermatt R, Zinkernagel AS, et al. Endothelial cell infection and endotheliitis in COVID-19. Lancet. 2020 May;395(10234):14178.

18 Ronco C, Bellomo R, Kellum JA. Acute kidney injury. Lancet. 2019 Nov;394(10212): 1949-1964.

19 Husain-Syed F, Wilhelm J, Kassoumeh S, Birk H-W, Herold S, Vadász I, et al. Acute kidney injury and urinary biomarkers in hospitalized patients with coronavirus disease 2019. Nephrol Dial Transplant. 2020. 10.1093/ndt/ gfaal62.

20 Moore JB, June CH. Cytokine release syndrome in severe COVID-19. Science. 2020 May;368(6490):473-4.

21 Monteleone G, Sarzi-Puttini PC, Ardizzone S. Preventing COVID-19-induced pneumonia with anticytokine therapy. Lancet Rheumatol. 2020 May;2(5):e255-6.

22 Zhong J, Tang J, Ye C, Dong L. The immunology of COVID-19: is immune modulation an option for treatment? Lancet Rheumatol. 2020 May;2(7):e428-36.

23 Huet $\mathrm{T}$, Beaussier $\mathrm{H}$, Voisin O, Jouveshomme S, Dauriat G, Lazareth I, et al. Anakinra for severe forms of COVID-19: a cohort study. Lancet Rheumatol. 2020 May;2(7):e393-400.

24 Tay MZ, Poh CM, Rénia L, MacAry PA, Ng LFP. The trinity of COVID-19: immunity, inflammation and intervention. Nat Rev Immunol. 2020 Jun;20(6):363-74.

25 Ronco C, Reis T, Husain-Syed F. Management of acute kidney injury in patients with COVID-19. Lancet Respir Med. 2020 May.
26 Berlot G, Tomasini A, Pognuz ER, Randino A, Chiella F, Fata CL, et al. The combined use of tocilizumab and hemoadsorption in a patient with COVID-19-associated pneumonia: case report. Nephron. 2020 Jul.

27 Ronco C, Marchionna N, Brendolan A, Neri M, Lorenzin A, Martínez Rueda AJ. Expanded haemodialysis: from operational mechanism to clinical results. Nephrol Dial Transplant. 2018 Oct;33(Suppl 3):iii41-7.

28 Krishnasamy R, Hawley CM, Jardine MJ, Roberts MA, Cho Y, Wong M, et al. A trial evaluating mid cut-off value membrane clearance of albumin and light chains in hemodialysis patients: a safety device study. Blood Purif. 2020 Jan;1-11.

29 Wolley M, Jardine M, Hutchison CA. Exploring the clinical relevance of providing increased removal of large middle molecules. Clin J Am Soc Nephrol. 2018 May;13(5):80514.

30 Wolley MJ, Hutchison CA. Large uremic toxins: an unsolved problem in end-stage kidney disease. Nephrol Dial Transplant. 2018 Oct; 33(Suppl 1_3):iii6-11.

31 Cozzolino M, Magagnoli L, Ciceri P, Conte F, Galassi A. Effects of a medium cut-off (Theranova ${ }^{\oplus}$ ) dialyser on haemodialysis patients: a prospective, cross-over study. Clin Kidney J. 2019 Nov.

32 Saran R, Robinson B, Abbott KC, Agodoa LY, Albertus P, Ayanian J, et al. US renal data system 2019 annual data report: epidemiology of kidney disease in the United States. Am J Kidney Dis. 2020 Jan;75(1):A6-7.

$33 \mathrm{Wu}$ J, Li J, Zhu G, Zhang Y, Bi Z, Yu Y, et al. Clinical features of maintenance hemodialysis patients with 2019 novel coronavirus-infected pneumonia in Wuhan, China. Clin J Am Soc Nephrol. 2020 May:CJN.04160320.

34 Zickler D, Schindler R, Willy K, Martus P, Pawlak M, Storr M, et al. Medium cut-off (MCO) membranes reduce inflammation in chronic dialysis patients-a randomized controlled clinical trial. PLoS One. 2017 Jan; 12(1):e0169024.

35 Belmouaz M, Bauwens M, Hauet T, Bossard V, Jamet P, Joly F, et al. Comparison of the removal of uraemic toxins with medium cutoff and high-flux dialysers: a randomized clinical trial. Nephrol Dial Transpl. 2019 Oct; 35(2):328-35.

36 Ronco C, Clark WR. Haemodialysis membranes. Nat Rev Nephrol. 2018 Jun;14(6): 394-410. 